\title{
Mechanistic Coupling of Dislocation and Shear Transformation Zone Plasticity in Crystalline-Amorphous Nanolaminates
}

\author{
Bin Cheng and Jason R. Trelewicz* \\ Department of Materials Science and Engineering, Stony Brook University, Stony Brook, NY 11794 \\ *Corresponding Author at Stony Brook University: jtrelewicz@stonybrook.edu
}

\begin{abstract}
The deformation behavior of crystalline-amorphous nanolaminates is explored through molecular dynamics simulations using nanolaminate models that contain columnar nanograins in the crystalline layers to more closely resemble experimentally accessible nanolaminate structures. Quantitative analysis of the plastic strain distribution among competing mechanisms and their coupling at the nanoscale is accomplished through the implementation of continuum deformation metrics. The transfer of plastic strain between shear transformation zone (STZ) and dislocation plasticity initially transpires through the emission of dislocations from STZ activity impinging on the amorphous-crystalline interface (ACI). The addition of grain boundaries biases this process to regions near the boundaries at low strains, which reduces the activation barrier for the onset of dislocation plasticity. With increasing strain, dislocations are absorbed into the amorphous layers via slip transfer across the ACI, in turn triggering the activation of new STZs. Cooperative slip transfer between dislocations and STZs suppresses grain boundary microcracking collectively with large-scale shear localization, and provides an explanation for the enhanced toughness of crystalline-amorphous nanolaminates.
\end{abstract}

Keywords: Crystalline-amorphous nanolaminates, Nanocrystalline metals, Molecular dynamics, Grain boundaries, Amorphous-crystalline interface 


\section{Introduction}

The mechanical behavior of nanocrystalline metals has been studied extensively over the past decade to understand the fundamental physics underpinning their remarkable strength and unusual deformation physics [1-6]. The illustrious Hall-Petch scaling law, which describes the increase in strength with grain size reduction $[7,8]$, captures strength scaling well into the nanocrystalline regime and is a consequence of grain boundaries inhibiting the mobility of dislocations [9, 10]. However, at grain sizes of about 10-20 nm, the Hall-Petch relationship breaks down [5, 11, 12] and classical dislocation dynamics shift to grain boundary-mediated dislocation processes [13-15]. The net accumulation of dislocations at grain boundaries during deformation often leads to grain boundary microcracking [6], which limits the ductility of nanocrystalline metals to about a few percent tensile elongation to failure [3, 16].

One promising route to improving the ductility of nanocrystalline metals is using gradient nanostructures containing, e.g., bimodal nanocrystalline grain size distributions [17] or periodically modulated crystalline-amorphous patterned structures [18]. While amorphous metals also suffer from limited plastic strain prior to fracture as a result of plastic strain localizing to form shear bands [19-21], a number of observations have suggested that the synergistic interaction between dislocation and shear transformation zone (STZ) plasticity across the amorphous-crystalline interface (ACI) can fundamentally alter the mechanical behavior [18, 22-25]. These materials represent a specific subset of metallic multilayers often referred to as crystalline-amorphous nanolaminates, and have demonstrated the soughtafter combination of high strength $(>1.0 \mathrm{GPa})$ and improved tensile elongation to failure $(>13 \%)$ that has eluded equiaxed nanocrystalline metals for over a decade [18, 22, 26-31].

In light of the critical role grain boundaries play in the deformation physics of nanocrystalline metals, the introduction of incoherent interfaces (i.e. ACIs) can be expected to open up new regimes in the 
mechanical behavior. In equiaxed nanocrystalline Ni-W alloys, Rupert et al. [32] demonstrated that through manipulation of the interfacial state, defect sources can be limited for enhancing strength without augmenting the fundamental mechanisms accommodating plasticity. It is in this vein that ACIs have been employed to engineer sources and sinks for defects in crystalline-amorphous nanolaminates, and a pioneering example is found in the $\mathrm{Cu}-\mathrm{Cu}_{3} \mathrm{Zr}$ system studied by Wang et al.[18]. These materials exhibited exceptional ductility relative to both crystalline metallic multilayers and equiaxed nanocrystalline $\mathrm{Cu}$ while retaining a yield strength of $\sim 1 \mathrm{GPa}$. Measurements of the activation volume and strain rate sensitivity were consistent with interface-mediated dislocation plasticity [27], and substantiated post-mortem transmission electron microscopy (TEM) observations.

Several additional studies have demonstrated ductility improvements in crystalline-amorphous nanolaminates citing comparisons with equiaxed nanocrystalline metals $[31,33,34]$ while others have focused on understanding the implications of the hierarchical structure for limiting shear localization in the amorphous regions [22]. Introduction of the nanocrystalline layers inherently disrupts the continuity of the amorphous phase, which has been demonstrated to constrain shear band growth [35] and promote homogenous plastic flow [36]. Simultaneously, the inherent structural length scale defined by the thickness of the amorphous layers suppresses the onset of shear instabilities. Citing the agedrejuvenation-glue-liquid shear band model [37], Wang et al. suggested that shear localization was inhibited when the amorphous layer thickness was less than a critical incubation length scale required for STZs to evolve into a mature shear band [18]. Despite conceptual differences in the synergistic effects of yielding in each phase, a number of studies [38-40] have substantiated the embryonic shear band argument for suppressing shear instabilities in crystalline-amorphous nanolaminates.

Significant insight has been gained on the coupling between dislocation and STZ plasticity through compression and nanoindentation experiments, which facilitate plastic strain accumulation without the onset of early fracture that results from localization in tension. By analyzing the plastic zone 
beneath the indents via correlative atom probe tomography and TEM, Guo et al. determined that dislocation glide in the crystalline $\mathrm{Cu}$ layers coincided with shear banding in the $\mathrm{CuZr}$ amorphous layers [24, 25, 41]. Microcompression testing of $\mathrm{Cu} / \mathrm{CuZr}$ nanolaminates by Zhang et al. [23, 42] revealed that dislocation-free nanocrystalline layers were accompanied by the nucleation of nanocrystallites within the amorphous layers. This deformation-induced partial devitrification was attributed to the transfer of plastic strain upon the absorption of dislocations across the ACI into the amorphous layers. Despite these compelling observations, assessment of the mechanistic coupling between dislocation and STZ plasticity has been largely based on inferences from post-mortem analysis of defects or other local structural changes.

Atomistic nanolaminate models are particularly convenient for direct investigation of deformation mechanisms as a function of the hierarchical structural length scales and deformation conditions (e.g. temperature, strain rate, loading direction) [43]. The triggering of STZs upon the absorption of dislocations in the amorphous layers was demonstrated in the uniaxial tensile simulations conducted by Wang et al. [18] on nanolaminates containing single crystal $\mathrm{Cu}$ layers. This finding was further substantiated in the simulations by Pan and Rupert [44] on bicrystals containing amorphous intergranular films, which acted as efficient sinks for dislocations that delayed the onset of interfacial cracking relative to "clean" grain boundaries. Brandl et al. [28] explored $\mathrm{Cu}-\mathrm{CuZr}$ crystalline-amorphous nanolaminates comparable to the previously described simulation structures under shear deformation boundary conditions. Interfacial shear was accommodated by the emission and propagation of dislocations at the $\mathrm{ACI}$, in turn facilitating co-deformation of the nanolaminate phases. Interfacial sliding has also been connected to localized STZ plasticity acting in cooperation with dislocation motion by Chen et al. [45]. Finally, the addition of free surfaces under simulated compressive loading by Arman et al. [46] revealed that dislocations, particularly of screw orientation, nucleated from the free surfaces but were subsequently absorbed at the ACIs. 
The coupling between dislocation and STZ plasticity at the ACIs in crystalline-amorphous nanolaminates provides a new mechanism for accommodating plastic strain during deformation. While several pioneering experiments have demonstrated the unique combination of strength and ductility afforded by the co-deformation of the crystalline and amorphous phases, mechanistic insights have largely been inferred from post-mortem deformation structures. Molecular dynamics simulations have been successful in capturing transient coupling events between the disparate deformation mechanisms operating at the ACIs. Despite these perceptive results, a number of open questions must be addressed to bridge the deformation physics from simulations with experimental mechanical behavior measurements. Perhaps one of the most important issues is that the existing computational models employ single crystal orientations completely absent of grain boundaries, which represent a critical feature in the deformation of nanostructured metals. How will grain boundaries bias the onset of dislocation and STZ plasticity in the respective regions of the nanolaminate, and what are their implications for the interfacial deformation physics? With a better understanding of the synergistic interactions between grain boundaries, ACIs, and the mechanisms accommodating plastic strain at the nanoscale, nanolaminate architectures can be designed to expand the strength-ductility envelope in nanostructured metals.

In this paper, a new crystalline-amorphous nanolaminate model is constructed that incorporates grain boundaries through the addition of columnar nanocrystalline grains in the crystalline layers to more closely resemble experimentally accessible nanolaminate structures. Uniaxial tensile deformation is accomplished using molecular dynamics with a strain rate of $10^{8} \mathrm{~s}^{-1}$, and the strain distribution among competing mechanisms is quantified via continuum deformation metrics. Our results demonstrate that STZ activity at the ACI is influenced by the presence of grain boundaries and responsible for the emission of interfacial dislocations, which are absorbed collectively by grain boundaries and the amorphous layers through continued deformation. Dislocation annihilation at the ACIs combined with a reduction in grain boundary plasticity abates grain boundary microcracking, thus providing an explanation for the 
exceptional ductility exhibited by crystalline-amorphous nanolaminates relative to equiaxed nanocrystalline counterparts.

\section{Simulation Methods}

Molecular dynamics (MD) simulations were carried out using the Large-scale Atomic/Molecular Massively Parallel Simulator (LAMMPS) platform [47]. The embedded-atom method (EAM) potential developed by Mendelev et al. [48] for the $\mathrm{Cu}-\mathrm{Zr}$ binary system was employed, as it provides a good representation for both $\mathrm{Cu}-\mathrm{Zr}$ metallic glasses [48] and pure crystalline $\mathrm{Cu}$ [49]. To build the various nanolaminate configurations, a $\mathrm{Cu}_{64} \mathrm{Zr}_{36}$ amorphous alloy was generated by heating a single crystal $\mathrm{Cu}-\mathrm{Zr}$ alloy to $2000 \mathrm{~K}$ for $2 \mathrm{~ns}$, followed by quenching to $300 \mathrm{~K}$ at a cooling rate of $0.01 \mathrm{~K} \cdot \mathrm{ps}^{-1}$. The single crystal-amorphous nanolaminate (SC-NL) was then constructed by combining a $5 \mathrm{~nm}$-thick layer of the quenched $\mathrm{Cu}_{64} \mathrm{Zr}_{36}$ amorphous alloy with a $10 \mathrm{~nm}$-thick single crystal $\mathrm{Cu}$ layer oriented with the [11̄ $]$, [110], and [111] directions along the $\mathrm{x}, \mathrm{y}$ and $\mathrm{z}$ directions, respectively. The application of periodic boundary conditions and homogenous structure effectively resulted in this configuration requiring only 11,930 atoms to model a 5/10 (i.e. a $5 \mathrm{~nm}$-thick amorphous layer and $10 \mathrm{~nm}$-thick crystalline layer) single crystal-amorphous nanolaminate. The SC-NL is illustrated in Figure 1a, and is comparable to the structure studied by Wang et al. [18].

A second crystalline-amorphous nanolaminate configuration was constructed for this study where columnar nanocrystalline grains were introduced into the crystalline layer, as shown in Figure $1 \mathrm{~b}$. This columnar nanocrystal-amorphous nanolaminate (CN-NL) structure is a better representation of experimentally attainable nanolaminates that contain grain boundaries in the crystalline layers, and employed the same 5/10 configuration as the SC-NL. A plan view of the $10 \mathrm{~nm}$-thick columnar nanocrystalline layer is illustrated in the inset of Figure $1 \mathrm{~b}$, where the $10 \mathrm{~nm}$ in-plane grain size was achieved by applying a Voronoi tessellation method [50] in the $x-y$ plane only; in the z-direction, the 
grains were constructed to produce a $10 \mathrm{~nm}$-thick columnar structure that terminates at the ACI. As demonstrated by the initial grain size distribution in Figure 2a, the Voronoi structure contained a number of both large $(>20 \mathrm{~nm})$ and small $(<5 \mathrm{~nm})$ grains relative to the average grain size. To sharpen the grain size distribution and eliminate outlying grain sizes, a Monte Carlo algorithm was applied to achieve a minimum grain size of $5 \mathrm{~nm}$ and a larger frequency of grains near the mean grain size of $10 \mathrm{~nm}$; this optimized distribution is also depicted in Figure 2a, and exhibited a volume-averaged grain size of 11.4 $\mathrm{nm}$.

The initial misorientation distribution in $\mathrm{x}-\mathrm{y}$ plane of the CN-NL structure also contained a large number of low-angle misorientations $\left(<14^{\circ}\right)$ as depicted in Figure 2b, which will undergo grain coalescence via grain boundary migration [51] resulting in an increase in the mean grain size. To eliminate these mobile low-angle grain boundaries, a second Monte Carlo algorithm was applied to the $\mathrm{CN}-\mathrm{NL}$ structure to maximize the average misorientation angle from $27.91^{\circ}$ to $38.04^{\circ}$ while simultaneously reducing the number of low-angle grain boundaries (cf. Figure 2b). A total of 25 grains was used in the construction of the columnar nanocrystalline layer, and when combined with the $\mathrm{Cu}_{64} \mathrm{Zr}_{36}$ amorphous layer, the optimized 5/10 CN-NL structure contained a total of 2,211,972 atoms for a sample size of $44.4 \mathrm{~nm} \times 44.4 \mathrm{~nm} \times 14.8 \mathrm{~nm}$.

A final equiaxed nanocrystalline $\mathrm{Cu}(\mathrm{NC}-\mathrm{Cu})$ sample was constructed using a three-dimensional Voronoi tessellation method to provide a direct comparison with the two nanolaminate structures. In particular, the $\mathrm{NC}-\mathrm{Cu}$ simulation cell contained 25 grains with a volume-averaged mean grain size and misorientation angle of $11.4 \mathrm{~nm}$ and $38.04^{\circ}$, respectively. These parameters were selected to match the volume-averaged mean grain size and misorientation distribution of the crystalline layer in the CN-NL sample. The resulting NC-Cu structure, which contained 1,622,110 atoms and a cell size of $27.09 \mathrm{~nm} \times$ $27.07 \mathrm{~nm} \times 27.07 \mathrm{~nm}$, is illustrated in Figure 1c. 
An energy minimization procedure was performed on all three structures to reach a final relative energy convergence of $10^{-12}$, followed by a simulated anneal at $650 \mathrm{~K}$ for $1 \mathrm{~ns}$ to generate realistic ACIs. This temperature was carefully chosen to provide sufficient thermal energy for local relaxation of the interfaces while remaining well below the glass transition temperature of $737 \mathrm{~K}$ for the $\mathrm{Cu}_{64} \mathrm{Zr}_{36}$ amorphous alloy [52]. The structures were then cooled to a temperature of $300 \mathrm{~K}$ using a rate of $1 \mathrm{~K} \cdot \mathrm{ps}^{-1}$ and relaxed for an additional $0.4 \mathrm{~ns}$ to eliminate the pressure in all dimensions. The deformation simulations were conducted at $300 \mathrm{~K}$ using uniaxial loading in the $\mathrm{x}$-direction at a constant strain rate of $10^{8} \cdot \mathrm{s}^{-1}$, and the normal stresses in the $\mathrm{y}$ - and $\mathrm{z}$-directions were relaxed to zero to allow lateral contraction during deformation. An isobaric-isothermal (NPT) ensemble was used to control the temperature and pressure during relaxation and deformation, and all simulations employed a time-step of $2 \mathrm{fs}$.

Characterization of the structures employed multiple quantitative tools often beginning with a common neighbor analysis (CNA) method [53, 54], which indexes each atom according to its local atomic coordination. The structures in Figure 1 employ the CNA scheme, where green atoms exhibit face-centered cubic (FCC) coordination, red atoms hexagonal close-packed (HCP) coordination (e.g. atoms commonly found in stacking faults), and grey atoms other coordination; the latter generally captures atoms occupying grain boundary sites in the crystalline layer and all atoms in the amorphous layer. Following deformation, local atomic slip was quantified using a slip vector analysis [55] with an atom's slip vector, $\boldsymbol{s}^{\alpha}$, defined as:

$$
\boldsymbol{s}^{\alpha}=-\frac{1}{n_{s}} \sum_{\beta \neq \alpha}^{n}\left(\boldsymbol{x}^{\alpha \beta}-\boldsymbol{X}^{\alpha \beta}\right)
$$

where $n$ is atom $\alpha$ 's total number of nearest neighbors and $n_{s}$ is atom $\alpha$ 's total number of slipped nearest neighbors. The variables $\boldsymbol{x}^{\alpha \beta}$ and $\boldsymbol{X}^{\alpha \beta}$ represent the interatomic distance vectors between atom $\alpha$ and neighbor atom $\beta$ in a deformed condition and reference state, respectively. 
Combined with CNA values, the slip vector can be used to estimate the Burger's vector of each atom and distinguish between competing deformation mechanisms [56]. In the subject simulations, particular emphasis was placed on delineating partial from full dislocation slip. Partial dislocations containing HCP coordinated atoms in a stacking fault will have a slip vector approximately equal to the Burger's vector for partial slip, i.e. $\left\|\boldsymbol{b}_{\boldsymbol{p}}\right\|=a / \sqrt{6}$ where a is the lattice constant. Full dislocation slip will instead involve FCC atoms, which exhibit a slip vector comparable to the Burger's vector for full slip, i.e. $\left\|\boldsymbol{b}_{\boldsymbol{f}}\right\|=a / \sqrt{2}$. For $\mathrm{Cu}$ with a lattice constant of $3.615 \AA$, the slip vectors for partial and full slip will be approximately 1.47 and $2.55 \AA$, respectively. The slip vector was also employed to quantify local deformation for grain boundary and amorphous atoms, which enabled a quantitative comparison between competing deformation mechanisms in the crystalline and amorphous layers [55].

While the slip vector is convenient for identifying deformation mechanisms and their relative distributions, the contribution of these mechanisms to the total strain was captured using the Green strain tensor, which is defined as:

$$
E=\frac{1}{2}\left[F^{T} F-I\right]
$$

where $\boldsymbol{I}$ is the identity tensor and $\boldsymbol{F}$ is the deformation gradient calculated according to the method described by Tucker et al. [56]. The application of continuum-level kinematic metrics such as the Green strain tensor enabled a quantitative characterization of strain accommodation in the subject nanolaminate structures, thus representing an important tool for uncovering the deformation physics at the nanoscale. Finally, to study the implications of various deformation mechanisms for void formation, a surface mesh method was employed to calculate void volume fractions formed during uniaxial deformation. The opensource OVITO software [57] was used in the CNA and surface mesh techniques, whereas the slip vector and Green strain tensor were determined using custom codes designed for execution in C; all analysis codes are available from the authors upon request. 


\section{Deformation Mechanisms and Strain Accommodation}

In this section, the deformation behavior of the three simulation structures are presented focusing first on the flow curves, followed by a description of the underlying mechanisms accommodating plasticity, and concluding with the distribution of strain among these competing deformation mechanisms. The stress-strain curves for the SC-NL, CN-NL and NC-Cu structures deformed under uniaxial tensile loading at $300 \mathrm{~K}$ with a strain rate of $10^{8} \cdot \mathrm{s}^{-1}$ are shown in Figure 3. While few disparities were apparent in the flow curves for the NC-Cu and CN-NL structures, the SC-NL sample exhibited noticeably different yielding behavior as evidenced by the abrupt stress drops with increasing strain. This was also manifested in the mechanical properties cataloged in Table 1, where the elastic modulus and stress at the onset of global plasticity (determined using a $0.2 \%$ offset criterion) of the SC-NL were significantly greater relative to the equiaxed nanocrystalline and columnar nanolaminate structures.

While the reduced elastic modulus of the SC-NL structure can be attributed to the anisotropy of the system and specific crystallographic orientation aligned with the tensile axis [58], additional analysis was required to understand the mechanisms responsible for the onset of global plasticity. Deformation snapshots were constructed as a function of the applied strain with emphasis herein placed on differentiating mechanisms in the SC-NL and CN-NL structures; the deformation physics in NC-Cu are addressed extensively in literature $[56,59,60]$ and thus only those aspects pertinent to the discussion of toughness are presented in Section 5. Snapshots are shown at three different strains in Figure 4 for the SN-NL structure with atoms colored by both the CNA value and slip vector.

Local plasticity initiated in the SC-NL at a strain of approximately $3 \%$ that derived from the activation of STZs in the amorphous layers. The onset of global plasticity corresponding to the appreciable stress drop of greater than $3.5 \mathrm{GPa}$ at approximately $6 \%$ strain was attributed to the emission of a dislocation from the lower ACI as evidenced in the CNA representation of Figure 4 viewed along the [110] direction; the leading partial (grey 'other coordination' atoms) and stacking fault (red HCP- 
coordinated atoms) emerged just prior to $6.6 \%$ strain. Atoms in the stacking fault have a characteristic slip vector of $1.4-1.6 \AA[55,56]$, and partial dislocation activity was thus captured by the green atoms in the slip vector snapshots. A major advantage of employing the slip analysis is its ability to capture full dislocation plasticity $[55,56]$, which is absent in the CNA representation as fully slipped atoms return to their initial FCC configuration. For example, the leading partial dislocation emitted from the ACI at 6.6\% strain was followed by a trailing partial and absorption of this full dislocation at the adjacent ACI just prior to $7.2 \%$ strain. Because the atoms experienced full slip with a slip vector magnitude of approximately $2.55 \AA$, full dislocation slip was evident in the slip vector representation (i.e. by the atoms colored red). This is even more prevalent at a strain of $11 \%$ where multiple slip traces resulting from the propagation of full dislocations were apparent in the slip vector analysis. By correlating dislocation activity via slip vector with the flow curve in Figure 3, the sharp stress drops were attributed to the emission of partial dislocations from the ACIs.

The flow curve for the CN-NL structure was free of large stress oscillations, which indicates that the transition to dislocation plasticity was fundamentally altered by the introduction of grain boundaries. From the deformation snapshots shown in Figure 5, the onset of dislocation plasticity corresponded to the emission of a leading partial dislocation and stacking fault from the intersection between the upper ACI and grain boundary plane at approximately 3.2\% strain. The slip vector analysis revealed a region of high slip in the amorphous layer directly above this grain boundary-ACI intersection, suggesting STZ activity triggered emission of the observed partial. The full dislocation generated upon emission of the trailing partial was absorbed across the adjacent ACI at 7.2\% strain, which can only be observed in the slip vector scheme. Absorption of this dislocation triggered a series of atomic rearrangements via STZ activity in the amorphous layer to accommodate the transfer of plastic strain. From this region of locally high slip, a new partial dislocation was nucleated at $10.8 \%$ strain within the same grain, and the resultant stacking fault is indicated by the white arrow in Figure 5. The neighboring grain exhibited extensive plasticity, 
which produced intrinsic and extrinsic stacking faults as well as deformation twins; the latter is denoted by the yellow arrow. Collectively, these deformation processes align with the defects observed through post-mortem TEM measurements on $\mathrm{Cu}-\mathrm{Cu}_{3} \mathrm{Zr}$ crystalline-amorphous nanolaminates [18].

Although deformation was accommodated by dislocations and STZs in both the SC-NL and CNNL structures, the introduction of grain boundaries in the latter augmented the onset of global plasticity based on the coupling between these disparate mechanisms. During the early stages of deformation, localized slip was first activated in the amorphous layer and further biased to regions adjacent to the grain boundaries, which in turn triggered the emission of dislocations. Collective shearing enabled by the redistribution of free volume during the process of STZ formation [61, 62] has previously been linked to the emission of dislocations at ACIs [28]. However, the biasing of STZ activity to regions in direct vicinity of the grain boundaries indicates a reduced activation barrier resulted from the presence of these boundaries. While this likely derived from limited slip transfer between the grain boundaries and amorphous regions promoting the formation of stress concentrations near the interface, it decidedly shifted the onset of dislocation plasticity to lower strains relative to the SC-NL structure.

As compared to $\mathrm{NC}-\mathrm{Cu}$, the flow curve for the $\mathrm{CN}-\mathrm{NL}$ structure exhibited many similar features and consequently, the properties calculated from the respective stress-strain curves were nearly identical. However, the mechanisms accommodating strain at the nanoscale differed considerably. Detailed analysis of the deformation mechanisms in $\mathrm{NC}-\mathrm{Cu}$ has been the subject of other pioneering works [59, 60, 63]. Here, we focus on differentiating the dominant mechanisms accommodating strain in all three structures using the Green strain tensor analysis, and the results are summarized in Figure 6.

Deformation was accommodated in the CN-NL structure by four primary mechanisms: straining of the FCC lattice, dislocations, grain boundary mediated plasticity, and STZ activity in the amorphous layer. In the SC-NL captured in Figure 6a, grain boundaries were not available to absorb plastic strain energy and as a result, dislocations accommodated the majority of plastic strain upon global yielding with 
additional contributions from STZ activity in the amorphous layer. In the absence of an amorphous layer, plastic strain largely partitioned to the grain boundaries in $\mathrm{NC}-\mathrm{Cu}$ as evident in Figure 6c. Conversely, the presence of the amorphous layer in the CN-NL structure suppressed grain boundary plasticity by more than $50 \%$, which was manifested by the substantial reduction in the grain boundary Green strain in Figure 6b. The fraction of strain accommodated by dislocation plasticity was nearly identical for the $\mathrm{NC}-\mathrm{Cu}$ and CN-NL structures; however, the Green strain analysis does not capture implicit interactions between dislocation and STZ plasticity. Such an analysis necessitates tracking of the atomic slip vector over predefined strain intervals, and is the subject of the following section. Nevertheless, the results demonstrated that grain boundaries significantly altered the process of strain accommodation in crystalline-amorphous nanolaminate models, which produced conspicuous changes in their flow behavior.

\section{Quantifying STZ-Dislocation Coupling and the Onset of Plasticity}

A comprehensive analysis of the coupling between STZ and dislocation plasticity is presented in this section with particular focus on quantitative characterization through tracking the of atomic slip vector during an isolated dislocation event. Dislocations have been demonstrated to nucleate at the ACI near regions of high atomic slip associated with STZ activity. While the formation of STZs in the SC-NL was distributed along the entire ACI and in agreement with other studies [18, 28, 45], STZ activity was biased to the grain boundary intersections with the ACI in the CN-NL structure suggesting that shear band formation will align with the grain boundary network. Plan views of the amorphous layer in the x-y plane of the CN-NL sample are illustrated in Figure 7a at three different strains; the formation of a shear band nucleus was evident at the lowest strain of $4 \%$. A doubling of the strain was accompanied by the appearance of large secondary shear bands, which thickened as strain was further increased to $12 \%$. The plan view of the crystalline layer in the $x-y$ plane shown in Figure $7 b$ demonstrates that shear band formation aligned with the dislocation networks, which also appeared to be mediated by the grain 
boundaries. While the coupling between dislocations and STZ plasticity is implied by these observations, the sequence of the deformation events cannot be ascertained from the deformation snapshots alone.

To understand the onset of dislocation plasticity and its connection to shear localization at the ACI, an analysis technique was developed to map the average slip vector in discrete layers by dividing the nanolaminate structures evenly into 12 slices parallel to the plane of the ACI, as illustrated in Figures 8a and $8 \mathrm{~b}$ for the SC-NL and CN-NL, respectively. Each slice was assigned a number from $1-12$ where slices S1, S2, S11, and S12 were located in the amorphous layer while slices S3 - S10 were distributed across the crystalline layer. The average slip vector was computed for each slice and mapped as a function of slice number in Figures $8 \mathrm{c}$ and $8 \mathrm{~d}$ for the SC-NL and CN-NL, respectively.

At low strains up to $6 \%$ in the SC-NL, the average slip vector trends in Figure $8 \mathrm{c}$ demonstrated severe strain partitioning to the amorphous layer, which confirmed the previous observations from the Green strain analysis in Figure 6a. Between 6 and 8\% strain, the precipitous jump in the average slip vectors for the crystalline slices corresponded to the initial stress drop just after $6 \%$ strain in the flow curve (cf. Figure 3 ) and the onset of dislocation plasticity from the Green strain analysis. The next abrupt increase between 10 and 12\% strain aligned with the second large stress drop in the flow curve and another burst of dislocation activity. Throughout all stages of deformation, the average slip vector was greater in the amorphous layer; however, slices located directly adjacent to the ACI (i.e. S2 and S11) exhibited a peak in the slip vector at all strains, resulting in the formation of a strain gradient across the ACI. This gradient was most severe just prior to the onset of plasticity in the crystalline layer, and was subsequently relieved by dislocations accommodating a significant fraction of the plastic strain (40\% from the Green strain analysis in Figure 6a).

The sudden jumps in the slip vector trends for the crystalline region of the SC-NL were absent during deformation of the CN-NL as evident in Figure 8d, substantiating the more gradual onset of macroplasticity in the flow curve. The absence of slip vector bursts can be attributed to grain boundaries 
mediating plastic strain accumulation during the initial stages of deformation as quantified in the Green strain analysis shown in Figure 6b. This was manifested as a steady monotonic increase in the average slip vector across the crystalline layer, i.e. in slices S3 - S10, particularly at strains of 2, 4, and $6 \%$ in Figure 8d. Further contributing to this gradual increase was the onset of dislocation plasticity at a strain of 3\%, which was delayed to $7 \%$ strain in the SC-NL. Elimination of the sharp strain gradient at the ACI suggests that the grain boundaries reduce the activation barrier for dislocation nucleation, and is commensurate with the models proposed by Asaro and Suresh [64] for nanocrystalline metals.

While tracking of the average slip vector in discretized slices demonstrated biased slip to the amorphous layers at low strain, a focused analysis of a single dislocation nucleation and propagation event was performed to quantify the different stages of microplasticity in the CN-NL structure. The deformation snapshots illustrated in Figure 9 capture the lifetime of a dislocation that was emitted from a grain boundary-ACI intersection as viewed on the (1 $\overline{1} \overline{1})$ slip plane. In the snapshots colored according to the CNA value, a leading partial was first nucleated at $4 \%$ strain and followed by a stacking fault as the partial traversed the grain. At $4.18 \%$ strain, a trailing partial was emitted that removed the stacking fault as it propagated across the grain, and was subsequently absorbed just after $4.26 \%$ strain at the lower grain boundary-ACI intersection resulting in full dislocation glide.

The emission of this trailing partial from a different location on the ACI indicated that grain boundary-ACI intersections are not solely responsible for the onset of dislocation plasticity. Instead, dislocation emission events directly correlated with regions of high local atomic slip at the ACI. In the snapshots indexed by slip vector in Figure 9, emission of the trailing partial at $4.18 \%$ strain occurred where STZ activity impinged on the ACI, not at its intersection with the grain boundary. Thus, while grain boundaries reduce the activation barrier for dislocation nucleation as previously noted, the general emission of dislocations from regions of high local atomic slip indicates that slip transfer across the ACI governed dislocation nucleation in the CN-NLs. Slip biasing to atoms near the grain boundaries in the 
amorphous layers, especially at low strains, can be attributed to stress concentrations that form at the grain boundary-ACI intersection rather than deriving from mechanisms proposed for grain boundary-mediated plasticity in equiaxed nanocrystalline metals such as grain boundary sliding [64].

To capture instantaneous changes in the slip history and substantiate the proposed slip transfer mechanism in crystalline-amorphous nanolaminates, the average atomic slip vector of each slice was

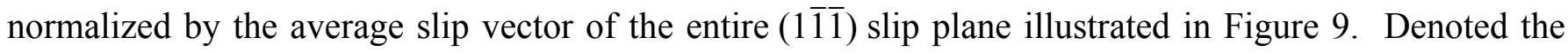
normalized average slip vector, trends are shown in Figure 10a for slices immediately adjacent to the upper ACI in the amorphous (S11) and crystalline (S10) layers as a function of strain; analogous results are shown in Figure 10b for slices adjacent to the lower ACI (S2 and S3, respectively).

Prior to emission of the leading partial, a strain gradient developed across the ACI, which is captured in Figure 10a by the enhanced normalized average slip vector for the amorphous atoms in slice $\mathrm{S} 11$ relative to the crystalline atoms in slice S10. At about 4\% strain, emission of the leading partial was accompanied by an abrupt drop in the normalized average slip vector of the amorphous S11 slice and immediately followed by a precipitous increase in the crystalline S10 slice. The second peak in the normalized average slip vector of the amorphous S11 slice around 4.18\% strain developed just prior to emission of the trailing partial, and was followed by the peak in the trend for the crystalline S10 slice as the trailing partial passed through this particular region of the nanolaminate. Emission of both the leading and trailing partials thus derived from an identical slip transfer mechanism where STZ activity impinging on the ACI produced regions of high local atomic slip, which in turn triggered the nucleation of dislocations to accommodate the high interfacial strain gradient.

Tracking of the normalized average slip vector in slices adjacent to the lower ACI revealed additional interactions between dislocations and STZ plasticity. Prior to the nucleation of the leading partial at the upper ACI, a strain gradient also developed at the lower ACI. However, emission of the leading partial manifested instead as a sharp drop in the normalized average slip vector in Figure 10b, 
which was a result of the overall increase in the entire slip plane's average slip vector upon nucleation of this partial dislocation. Propagation of the leading partial through the crystalline S3 slice occurred at approximately $4.1 \%$ strain, as evidenced by the abrupt increase in the normalized average slip vector of this region. Immediately following propagation through the crystalline S3 slice, the leading partial impinged on the lower ACI producing a peak in the normalized average slip vector of the amorphous S2 slice that corresponded to the activation of new STZs. Dislocation absorption by an amorphous phase has been demonstrated to trigger STZ activity in other studies [44, 65], and the results presented herein provide quantitative evidence for this slip transfer mechanism in crystalline-amorphous nanolaminates.

Direct coupling between dislocation slip and STZ plasticity has been shown to transpire through two mechanisms: (1) the emission of dislocations from sites of high local atomic slip deriving from STZ activity impinging on the ACI, and (2) the triggering of new STZs upon absorption of dislocations across an ACI. Once dislocation slip is established, these slip transfer mechanisms collectively operate throughout all stages of deformation and provide a sustainable process for accommodating plastic strain through coupled dislocation-STZ plasticity. Grain boundaries played a critical role in strain accommodation process especially the onset of global yielding, which resulted from biased dislocation emission from grain boundary-ACI intersections. These stages of deformation and the underlying mechanisms will therefore be more closely representative of the deformation behavior exhibited by experimental nanolaminates that inherently contain a hierarchy of structural length scales.

\section{Implications of Coupled Dislocation-STZ Plasticity for the Strength-Ductility Envelope}

The Green strain analysis summarized in Figure 6 provided quantitative evidence for plastic strain collectively partitioning to the grain boundaries and the amorphous layer prior to the onset of dislocation plasticity in the CN-NL structure. By tracking the atomic slip in discretized slices within a slip plane, a connection was established between grain boundary and STZ-mediated plasticity, which was further 
bridged to the process of dislocation nucleation that ultimately governed the strength of these materials. Conversely, ductility will be controlled by the ability to inhibit the formation of grain boundary microcracks while simultaneously circumventing strain localization in the amorphous regions. The role of dislocation-STZ coupling in impeding these failure mechanisms is addressed in this section, with a focus on slip transfer via dislocation absorption at the ACIs and its implications for shear localization and void formation.

Plastic strain accumulation in equiaxed nanocrystalline metals is often limited by the formation of stress concentrations that derive from dislocation-grain boundary interactions. These stress concentrations promote the initiation of grain boundary voids and cracks $[6,17]$, and their subsequent coalescence leads to brittle intergranular fracture [66]. To quantify the process of void formation, a surface meshing technique [57] was applied and the resulting void fractions are depicted in Figure 11a for each structure as a function of the applied strain. In the $\mathrm{NC}-\mathrm{Cu}$ and $\mathrm{CN}-\mathrm{NL}$ structures, void initiation occurred at approximately $6 \%$ strain, followed by growth at a constant rate up to $11 \%$ strain. A change in slope was apparent for $\mathrm{NC}-\mathrm{Cu}$ at $11 \%$ strain that captured a transition in the growth rate, which continued through the duration of the simulation. Consequently, the final void fraction in $\mathrm{NC}-\mathrm{Cu}$ at $20 \%$ strain was more than double the CN-NL structure. When combined with the complete absence of voids in the SCNL, these results indicate that void formation derived from the interaction of defects with grain boundaries.

In the context of the Green strain analysis in Figure 6, grain boundaries accounted for greater than $40 \%$ of the strain in $\mathrm{NC}-\mathrm{Cu}$, whereas only $20 \%$ was accommodated by grain boundaries in the CN-NL structure. However, $\mathrm{NC}-\mathrm{Cu}$ contained nearly double the volume fraction of grain boundaries $(16.6 \%)$ relative to the CN-NL structure $(8.7 \%)$, and grain boundaries will inherently accommodate a greater amount of plastic strain. This provides one possible explanation for the improved ductility of crystallineamorphous nanolaminates, i.e. they contain fewer grain boundary sites for the initiation of grain boundary 
microcracks. From a simple qualitative inspection, voids were determined to form in the CN-NL structure primarily at the grain boundaries upon impingement of a leading partial as demonstrated in Figure $11 \mathrm{~b}$ at $7 \%$ strain. This is in good agreement with equiaxed nanocrystalline metals where crack formation transpires preferentially at grain boundaries, [66] and is often linked to dislocation-grain boundary interactions [6]. Despite these similarities, the underlying mechanisms responsible for void initiation could also be influenced by the hierarchical structure albeit in a more inconspicuous manner.

A surface atom technique based on the distribution of Voronoi volumes was developed to quantify the correlation between void initiation sites and grain boundaries. The Voronoi volume employs nearest neighbor atoms to provide a local measure of the space occupied by each atom, and represents the volume of a Voronoi polyhedron that encompasses each atomic site [67]. The formation of a void produces a shift in some of an atom's nearest neighbors that manifests as increase in the average Voronoi volume. Consequently, atoms located on a void or crack surface denoted herein as "surface atoms" exhibit abnormally large Voronoi volumes greater than $18.5 \AA^{3}$, which lies convincingly outside the range of Voronoi volumes for $\mathrm{Cu}$ of $9-16 \AA^{3}$. Using this technique, surface atoms were identified and their allegiance to the grain interiors, grain boundaries, and ACIs tracked during deformation. The fraction of surface atoms determined for each of these regions is shown in Figure 11c as a function of the applied strain.

Surface atoms were detected in the grain boundary region at approximately $6 \%$ strain indicating that voids nucleated in the grain boundary regions (i.e. a $1.2 \mathrm{~nm}$ thick region around the grain boundaries) well after the early stages of plastic deformation involving dislocation, grain boundary, and STZ plasticity. With increasing strain, the fraction of surface atoms in the grain boundaries grew precipitously while it remained relatively insignificant in the intragranular regions. Only a small fraction of surface atoms were identified in the ACI region after about 9\% strain, which suggested that the ACIs reduced the propensity for void formation. This was substantiated by separating the void fractions for the ACI regions (i.e. slices 
S3 and S10) from the other crystalline slices of the CN-NL structure, and the trends are shown in Figure 11d. Voids initially formed in the interior crystalline slices as evident in the inset, and their volume fraction with increasing strain was consistently greater relative to the ACI regions. Taken collectively with the biased void nucleation within the grain boundary regions from Figure 11c, it can be inferred that voids formed predominantly in the central regions of grain boundaries.

Based on the above analysis, the ability of dislocations to be absorbed across the ACIs without the onset of void nucleation represents a new fundamental mechanism for limiting damage accumulation at the nanoscale. Our results are in excellent agreement with the atomistic simulations conducted by Pan and Rupert [44] that demonstrated delayed interfacial cracking upon the absorption of dislocations in boundaries containing amorphous complexions. From the alternative perspective of shear localization in monolithic amorphous alloys, the hierarchical structure of crystalline-amorphous nanolaminates confines shear localization within the thin amorphous layers. Limiting the propagation of a dominant shear front while simultaneously nucleating new shear bands through the transfer of plastic strain upon dislocation absorption provides two additional mechanisms for enhancing plastic strain accommodation, and in turn, ductility relative to monolithic metallic glass counterparts [40]. The unique ability to circumvent grain boundary microcracking collectively with catastrophic shear localization contributes to the appreciably improved toughness of crystalline-amorphous nanolaminates relative to equiaxed nanocrystalline metals.

\section{Conclusions}

In this study, molecular dynamics simulations were employed to quantify the plastic strain distribution among competing deformation mechanisms in crystalline-amorphous nanolaminate models containing columnar nanograins. Significant insight was gained into the coupling between disparate mechanisms at the nanoscale using continuum deformation metrics, summarized as follows: 
i. The addition of grain boundaries into the crystalline layers of the nanolaminate biased STZ activity to regions near these boundaries, which reduced the activation barrier for dislocation plasticity as manifested by the reduced stress at the onset of global yielding for the CN-NL.

ii. Direct coupling between dislocation slip and STZ plasticity transpired through two mechanisms including the emission of dislocations from sites of high local atomic slip deriving from STZ activity impinging on the ACI, and the triggering of new STZs upon absorption of dislocations across an ACI that in turn promotes additional dislocation plasticity.

iii. The biasing of void formation to the central regions of the grain boundaries relative to the ACIs and its connection to dislocation plasticity indicated that the accommodation of dislocations at ACIs abated void nucleation within the CN-NL structure.

iv. The propagation of a dominant shear front was limited by shear band confinement to the amorphous layers collectively with the nucleation of new shear bands promoted by the transfer of plastic strain upon dislocation absorption across the ACIs.

Based on the above results, the improved toughness of crystalline-amorphous nanolaminates relative to equiaxed nanocrystalline metals can be understood in the context of the interplay between disparate deformation mechanisms at the nanoscale and its ability to limit grain boundary microcracking while simultaneously inhibiting catastrophic shear localization. The incorporation of grain boundaries into the nanolaminate model was essential to capturing the influence of hierarchical structural length scales on the deformation physics, which drastically affected the damage accumulation mechanisms and flow behavior. Using these structural length scales to tune the plastic strain distribution among competing mechanisms, new design strategies can be realized for expanding the strength-ductility envelope in nanostructured metals. 


\section{Acknowledgements}

This work was supported by the National Science Foundation through Grant DMR-1410941. Atomistic simulations were conducted using resources of the Center for Functional Nanomaterials, which is a US DOE Office of Science Facility at Brookhaven National Laboratory under Contract No. DESC0012704. Results in this paper were also obtained using the high-performance computing system at the Institute for Advanced Computational Science at Stony Brook University.

\section{References}

[1] V. Yamakov, D. Wolf, S.R. Phillpot, A.K. Mukherjee, H. Gleiter. Deformation-mechanism map for nanocrystalline metals by molecular-dynamics simulation, Nature Materials 3 (2004) 43-47.

[2] T.G. Nieh, J. Wadsworth. Hall-Petch Relation in Nanocrystalline Solids, Scripta Metallurgica et Materialia 25 (1991) 955-958.

[3] M.A. Meyers, A. Mishra, D.J. Benson. Mechanical properties of nanocrystalline materials, Prog Mater Sci 51 (2006) 427-556.

[4] H. Conrad. Grain-size dependence of millimeters to nanometers, Metallurgical and Materials Transactions a-Physical Metallurgy and Materials Science 35A (2004) 2681-2695.

[5] J.R. Weertman. Hall-Petch Strengthening in Nanocrystalline Metals, Materials Science and Engineering a-Structural Materials Properties Microstructure and Processing 166 (1993) 161-167.

[6] K.S. Kumar, H. Van Swygenhoven, S. Suresh. Mechanical behavior of nanocrystalline metals and alloys, Acta Materialia 51 (2003) 5743-5774.

[7] E.O. Hall. The deformation and aging of mild steel III. Discussion of results Proc. Phys. Soc. B (UK) 64 (1951) 747-753.

[8] N.J. Petch. The upper yield stress of polycrystalline iron Acta Metallurgica 12 (1964) 59-65.

[9] X.L. Wu, E. Ma. Dislocations in nanocrystalline grains, Applied Physics Letters 88 (2006) -.

[10] M.A. Meyers, A. Mishra, D.J. Benson. The deformation physics of nanocrystalline metals: Experiments, analysis, and computations, Jom 58 (2006) 41-48.

[11] A.H. Chokshi, A. Rosen, J. Karch, H. Gleiter. On the Validity of the Hall-Petch Relationship in Nanocrystalline Materials, Scripta Metall Mater 23 (1989) 1679-1683.

[12] J.R. Trelewicz, C.A. Schuh. The Hall-Petch breakdown in nanocrystalline metals: A crossover to glass-like deformation, Acta Materialia 55 (2007) 5948-5958.

[13] V. Yamakov, D. Wolf, S.R. Phillpot, A.K. Mukherjee, H. Gleiter. Deformation mechanism crossover and mechanical behaviour in nanocrystalline materials, Philosophical Magazine Letters 83 (2003) 385-393.

[14] R.J. Asaro, P. Krysl, B. Kad. Deformation mechanism transitions in nanoscale fcc metals, Philosophical Magazine Letters 83 (2003) 733-743.

[15] E. Bitzek, C. Brandl, P.M. Derlet, H. Van Swygenhoven. Dislocation cross-slip in nanocrystalline fcc metals, Physical Review Letters 100 (2008) -.

[16] J.A. Sharon, H.A. Padilla, B.L. Boyce. Interpreting the ductility of nanocrystalline metals, Journal of Materials Research 28 (2013) 1539-1552.

[17] Y.M. Wang, M.W. Chen, F.H. Zhou, E. Ma. High tensile ductility in a nanostructured metal, Nature 419 (2002) 912-915. 
[18] Y.M. Wang, J. Li, A.V. Hamza, T.W. Barbee. Ductile crystalline-amorphous nanolaminates, Proceedings of the National Academy of Sciences of the United States of America 104 (2007) 1115511160.

[19] A.S. Argon. Plastic-Deformation in Metallic Glasses, Acta Metallurgica 27 (1979) 47-58.

[20] F. Spaepen. Microscopic Mechanism for Steady-State Inhomogeneous Flow in Metallic Glasses, Acta Metallurgica 25 (1977) 407-415.

[21] C.A. Schuh, T.C. Hufnagel, U. Ramamurty. Overview No.144 - Mechanical behavior of amorphous alloys, 55 (2007) 4067-4109.

[22] J.Y. Zhang, G. Liu, S.Y. Lei, J.J. Niu, J. Sun. Transition from homogeneous-like to shear-band deformation in nanolayered crystalline $\mathrm{Cu} /$ amorphous $\mathrm{Cu}-\mathrm{Zr}$ micropillars: Intrinsic vs. extrinsic size effect, Acta Materialia 60 (2012) 7183-7196.

[23] J.Y. Zhang, G. Liu, J. Sun. Self-toughening crystalline Cu/amorphous Cu-Zr nanolaminates: Deformation-induced devitrification, Acta Materialia 66 (2014) 22-31.

[24] W. Guo, E.A. Jägle, P.-P. Choi, J. Yao, A. Kostka, J.M. Schneider, D. Raabe. Shear-Induced Mixing Governs Codeformation of Crystalline-Amorphous Nanolaminates, Physical Review Letters 113 (2014) 035501.

[25] W. Guo, E. Jägle, J. Yao, V. Maier, S. Korte-Kerzel, J.M. Schneider, D. Raabe. Intrinsic and extrinsic size effects in the deformation of amorphous $\mathrm{CuZr} /$ nanocrystalline $\mathrm{Cu}$ nanolaminates, Acta Materialia 80 (2014) 94-106.

[26] J.J. Niu, P. Zhang, R.H. Wang, J.Y. Zhang, G. Liu, G.J. Zhang, J. Sun. Formation of multiple twins and their strengthening effect in nanocrystalline $\mathrm{Cu} / \mathrm{Zr}$ multilayer films, Materials Science and Engineering: A 539 (2012) 68-73.

[27] Y.M. Wang, A.V. Hamza, T.W. Barbee. Incipient plasticity in metallic glass modulated nanolaminates, Applied Physics Letters 91 (2007).

[28] C. Brandl, T.C. Germann, A. Misra. Structure and shear deformation of metallic crystallineamorphous interfaces, Acta Materialia 61 (2013) 3600-3611.

[29] M.C. Liu, J.C. Huang, Y.T. Fong, S.P. Ju, X.H. Du, H.J. Pei, T.G. Nieh. Assessing the interfacial strength of an amorphous-crystalline interface, Acta Materialia 61 (2013) 3304-3313.

[30] Y. Wang, J.Q. Zhou, S. Zhang, L. Wang. Mechanical analysis of the crystalline-amorphous laminated composite based on a two-phase model, Materials \& Design 51 (2013) 88-94.

[31] H.J. Pei, S.Y. Kuan, M.C. Liu, J.C. Huang. Tensile behavior of amorphous/nanocrystalline $\mathrm{ZrCu} / \mathrm{Cu}$ multilayered films with graded interfaces, Intermetallics 31 (2012) 191-195.

[32] T.J. Rupert, J.R. Trelewicz, C.A. Schuh. Grain boundary relaxation strengthening of nanocrystalline Ni-W alloys, Journal of Materials Research 27 (2012) 1285-1294.

[33] C.J. Lee, H.K. Lin, J.C. Huang, S.Y. Kuan. Tension behavior of free-standing amorphous film and amorphous-crystalline nanolaminates in submicron scale, Scripta Materialia 65 (2011) 695-698.

[34] F.F. Wu, K.C. Chan, S.T. Li, G. Wang, P. Lin. Tensile deformation of a Ti-based metallic glass composite lamella confined by commercially pure titanium, Philosophical Magazine Letters 94 (2014) 233-241.

[35] M.C. Liu, J.C. Huang, H.S. Chou, Y.H. Lai, C.J. Lee, T.G. Nieh. A nanoscaled underlayer confinement approach for achieving extraordinarily plastic amorphous thin film, Scripta Materialia 61 (2009) 840-843.

[36] M.C. Liu, X.H. Du, I.C. Lin, H.J. Pei, J.C. Huang. Superplastic-like deformation in metallic amorphous/crystalline nanolayered micropillars, Intermetallics 30 (2012) 30-34.

[37] F. Shimizu, S. Ogata, J. Li. Yield point of metallic glass, Acta Materialia 54 (2006) 4293-4298.

[38] T.G. Nieh, J. Wadsworth. Bypassing shear band nucleation and ductilization of an amorphouscrystalline nanolaminate in tension, Intermetallics 16 (2008) 1156-1159. 
[39] A. Donohue, F. Spaepen, R.G. Hoagland, A. Misra. Suppression of the shear band instability during plastic flow of nanometer-scale confined metallic glasses, Applied Physics Letters 91 (2007) 3.

[40] J.Y. Kim, D.C. Jang, J.R. Greer. Nanolaminates Utilizing Size-Dependent Homogeneous Plasticity of Metallic Glasses, Adv. Funct. Mater. 21 (2011) 4550-4554.

[41] W. Guo, J. Yao, E.A. Jägle, P.-P. Choi, M. Herbig, J.M. Schneider, D. Raabe. Deformation induced alloying in crystalline - metallic glass nano-composites, Materials Science and Engineering: A 628 (2015) 269-280.

[42] J.Y. Zhang, G. Liu, J. Sun. Crystallization-aided extraordinary plastic deformation in nanolayered crystalline $\mathrm{Cu} /$ amorphous Cu-Zr micropillars, Scientific Reports 3 (2013) 6.

[43] S. Yamamoto, Y.J. Wang, A. Ishii, S. Ogata. Atomistic Design of High Strength CrystallineAmorphous Nanocomposites, Materials Transactions 54 (2013) 1592-1596.

[44] Z. Pan, T.J. Rupert. Amorphous intergranular films as toughening structural features, Acta Mater 89 (2015) 205-214.

[45] K. Chen, S.-q. Shi, W. Zhu, X. Peng. Plastic deformation due to interfacial sliding in amorphous/crystalline nanolaminates, Computational Materials Science 109 (2015) 266-276.

[46] B. Arman, C. Brandl, S.N. Luo, T.C. Germann, A. Misra, T. Cagin. Plasticity in $\mathrm{Cu}(111) / \mathrm{Cu} 46 Z$ r54 glass nanolaminates under uniaxial compression, J. Appl. Phys. 110 (2011) 5.

[47] S. Plimpton. Fast Parallel Algorithms for Short-Range Molecular Dynamics, Journal of Computational Physics 117 (1995) 1-19.

[48] M.I. Mendelev, D.K. Rehbein, R.T. Ott, M.J. Kramer, D.J. Sordelet. Computer simulation and experimental study of elastic properties of amorphous $\mathrm{Cu}-\mathrm{Zr}$ alloys, Journal of Applied Physics 102 (2007).

[49] M.I. Mendelev, M.J. Kramer, C.A. Becker, M. Asta. Analysis of semi-empirical interatomic potentials appropriate for simulation of crystalline and liquid Al and Cu, Philos Mag 88 (2008) 1723-1750. [50] G. Voronoi. New applications of continuous parameters to the theory of quadratic forms. - First memoir - Some properties of perfect positive quadratic forms, J. Reine Angew. Math. 133 (1908) 97-178.

[51] A.J. Haslam, S.R. Phillpot, H. Wolf, D. Moldovan, H. Gleiter. Mechanisms of grain growth in nanocrystalline fcc metals by molecular-dynamics simulation, Materials Science and Engineering aStructural Materials Properties Microstructure and Processing 318 (2001) 293-312.

[52] N. Mattern, A. Schöps, U. Kühn, J. Acker, O. Khvostikova, J. Eckert. Structural behavior of CuxZr100-x metallic glass ( $\mathrm{x}=35-70)$, Journal of Non-Crystalline Solids 354 (2008) 1054-1060.

[53] D. Faken, H. Jónsson. Systematic analysis of local atomic structure combined with 3D computer graphics, Computational Materials Science 2 (1994) 279-286.

[54] H. Tsuzuki, P.S. Branicio, J.P. Rino. Structural characterization of deformed crystals by analysis of common atomic neighborhood, Computer Physics Communications 177 (2007) 518-523.

[55] J.A. Zimmerman, C.L. Kelchner, P.A. Klein, J.C. Hamilton, S.M. Foiles. Surface step effects on nanoindentation, Phys Rev Lett 87 (2001) 165507.

[56] G.J. Tucker, S. Tiwari, J.A. Zimmerman, D.L. McDowell. Investigating the deformation of nanocrystalline copper with microscale kinematic metrics and molecular dynamics, J Mech Phys Solids 60 (2012) 471-486.

[57] A. Stukowski. Visualization and analysis of atomistic simulation data with OVITO-the Open Visualization Tool, Model Simul Mater Sc 18 (2010).

[58] J. Schiotz, F.D. Di Tolla, K.W. Jacobsen. Softening of nanocrystalline metals at very small grain sizes, Nature 391 (1998) 561-563.

[59] H. Van Swygenhoven, P.A. Derlet. Grain-boundary sliding in nanocrystalline fcc metals, Physical Review B 64 (2001) 9.

[60] J. Schiotz, K.W. Jacobsen. A maximum in the strength of nanocrystalline copper, Science 301 (2003) 1357-1359. 
[61] A.S. Argon. Plastic deformation in metallic glasses, Acta Metallurgica 27 (1979) 47-58.

[62] A.R. Yavari, A.L. Moulec, A. Inoue, N. Nishiyama, N. Lupu, E. Matsubara, W.J. Botta, G. Vaughan, M.D. Michiel, Å. Kvick. Excess free volume in metallic glasses measured by X-ray diffraction, Acta Materialia 53 (2005) 1611-1619.

[63] H. Van Swygenhoven, P.M. Derlet, A.G. Froseth. Nucleation and propagation of dislocations in nanocrystalline fcc metals, Acta Materialia 54 (2006) 1975-1983.

[64] R.J. Asaro, S. Suresh. Mechanistic models for the activation volume and rate sensitivity in metals with nanocrystalline grains and nano-scale twins, Acta Materialia 53 (2005) 3369-3382.

[65] B. Arman, C. Brandl, S.N. Luo, T.C. Germann, A. Misra, T. Cagin. Plasticity in $\mathrm{Cu}(111) / \mathrm{Cu} 46 Z \mathrm{Z} 54$ glass nanolaminates under uniaxial compression, Journal of Applied Physics 110 (2011).

[66] K.S. Kumar, S. Suresh, M.F. Chisholm, J.A. Horton, P. Wang. Deformation of electrodeposited nanocrystalline nickel, Acta Materialia 51 (2003) 387-405.

[67] Y. Ritter, D. Sopu, H. Gleiter, K. Albe. Structure, stability and mechanical properties of internal interfaces in Cu64Zr36 nanoglasses studied by MD simulations, Acta Materialia 59 (2011) 6588-6593. 


\section{Tables}

Table 1: Mechanical properties of the SC-NL, CN-NL and NC-Cu structures.

\begin{tabular}{|c|c|c|c|}
\hline Structure & Elastic Modulus $(\mathrm{GPa})$ & $\begin{array}{c}\text { Strain at the Onset of } \\
\text { Plasticity (\%) }\end{array}$ & $\begin{array}{c}\text { Stress at the Onset of } \\
\text { Plasticity (GPa) }\end{array}$ \\
\hline SC-NL & 115.92 & 3.07 & 3.34 \\
\hline CN-NL & 103.46 & 2.38 & 2.26 \\
\hline NC-Cu & 98.52 & 2.2 & 1.97 \\
\hline
\end{tabular}




\section{Figures}

(a)

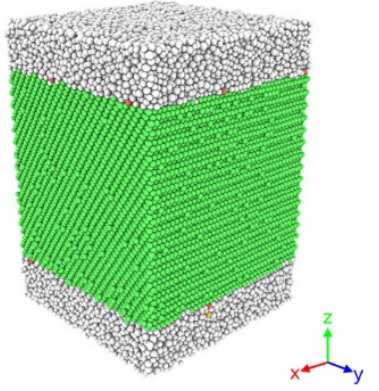

(b)

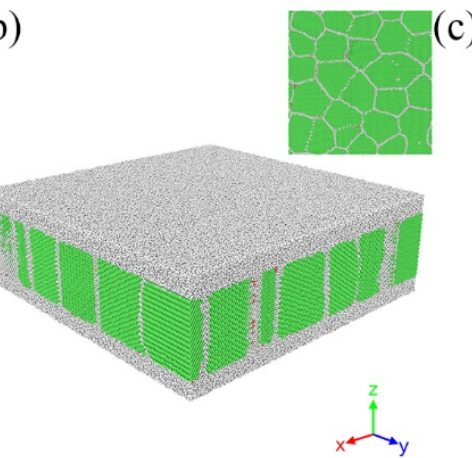

(c)

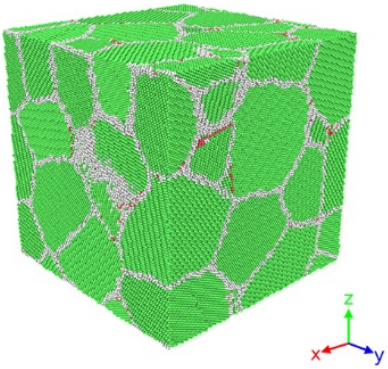

Figure 1: Initial nanolaminate structures consisting of a $5 \mathrm{~nm}$-thick $\mathrm{Cu}_{64} \mathrm{Zr}_{36}$ amorphous layer (shown divided into two $2.5 \mathrm{~nm}$-thick layers with periodic boundary conditions applied to the $\mathrm{x}$ y faces) combined with (a) a 10 nm-thick single crystal $\mathrm{Cu}$ layer with the [112] , [110] and [111] directions along the $\mathrm{x}, \mathrm{y}$ and $\mathrm{z}$ axes, respectively, and (b) a $10 \mathrm{~nm}$-thick columnar nanocrystalline $\mathrm{Cu}$ layer containing equiaxed grains in the $\mathrm{x}-\mathrm{y}$ plane as shown in the inset. (c) The equiaxed nanocrystalline structure containing 25 grains with a volume-averaged mean grain size of $11.4 \mathrm{~nm}$, which matches the CN-NL structure. Atoms are colored by CNA value, and the reader is referred to the web version of this article for interpretation of the references to color. 
(a)

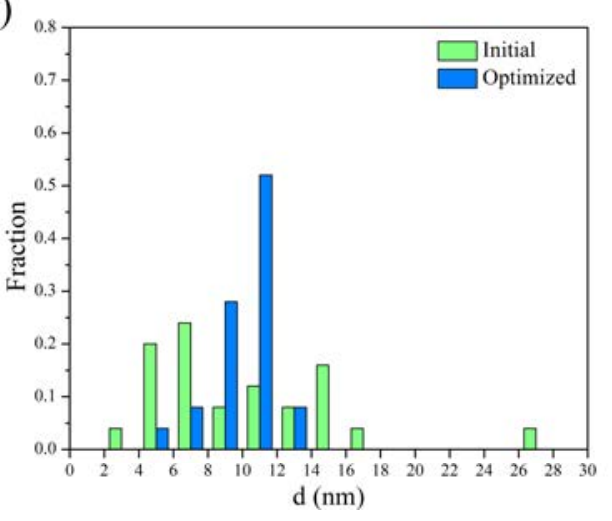

(b)

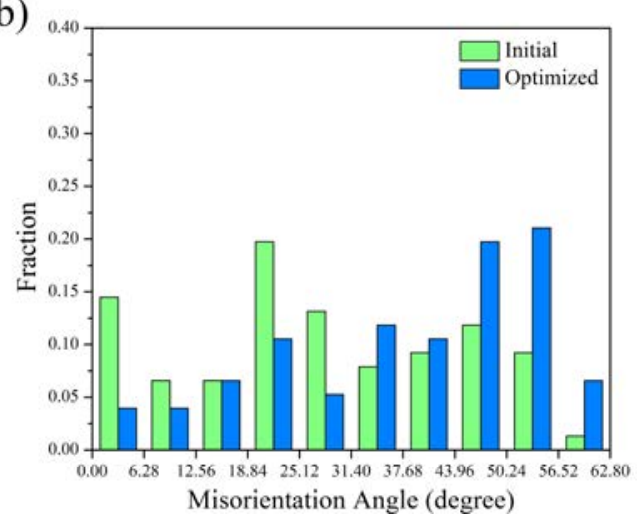

Figure 2: Structural characteristics of the CN-NL sample including (a) grain size and (b) misorientation distributions for both the initial and optimized configurations. The optimized structures were achieved through a Monte Carlo procedure to sharpen the grain size distribution and maximize the average misorientation angle. (For interpretation of the references to color in this figure legend, the reader is referred to the web version of this article.) 


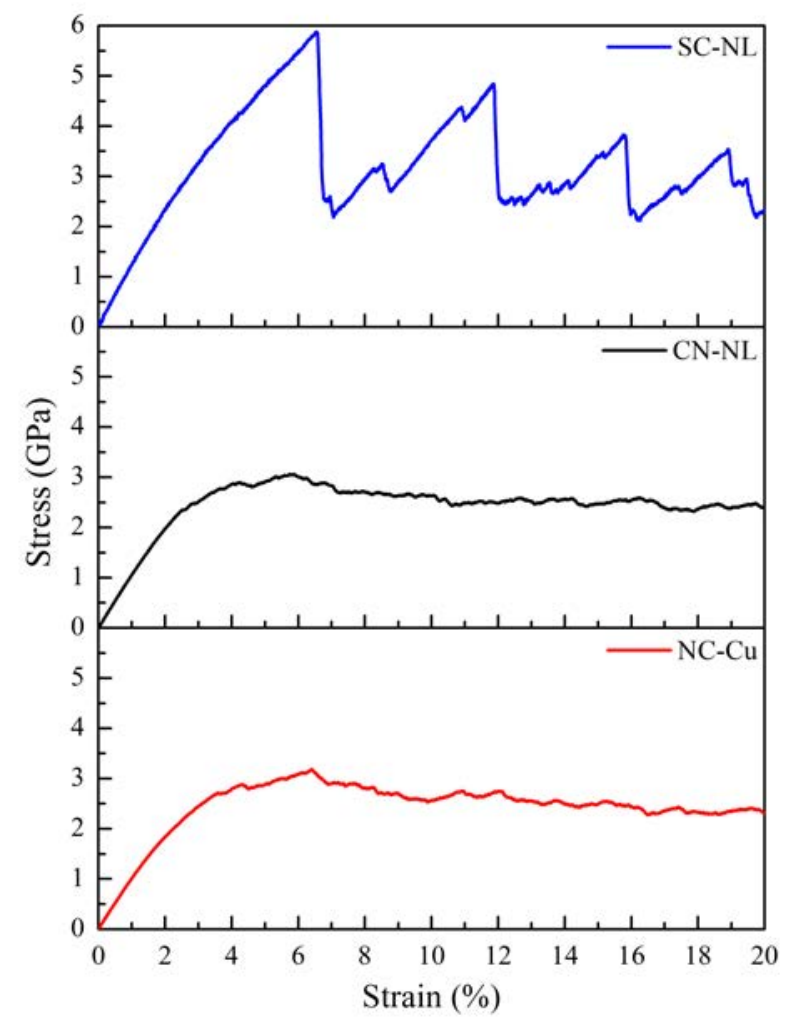

Figure 3: Flow curves for the single crystal-amorphous nanolaminate (SC-NL), columnar nanocrystal-amorphous nanolaminate $(\mathrm{CN}-\mathrm{NL})$, and equiaxed nanocrystalline $\mathrm{Cu}(\mathrm{NC}-\mathrm{Cu})$ samples. Mechanical properties determined from these data can be found in Table 1. 

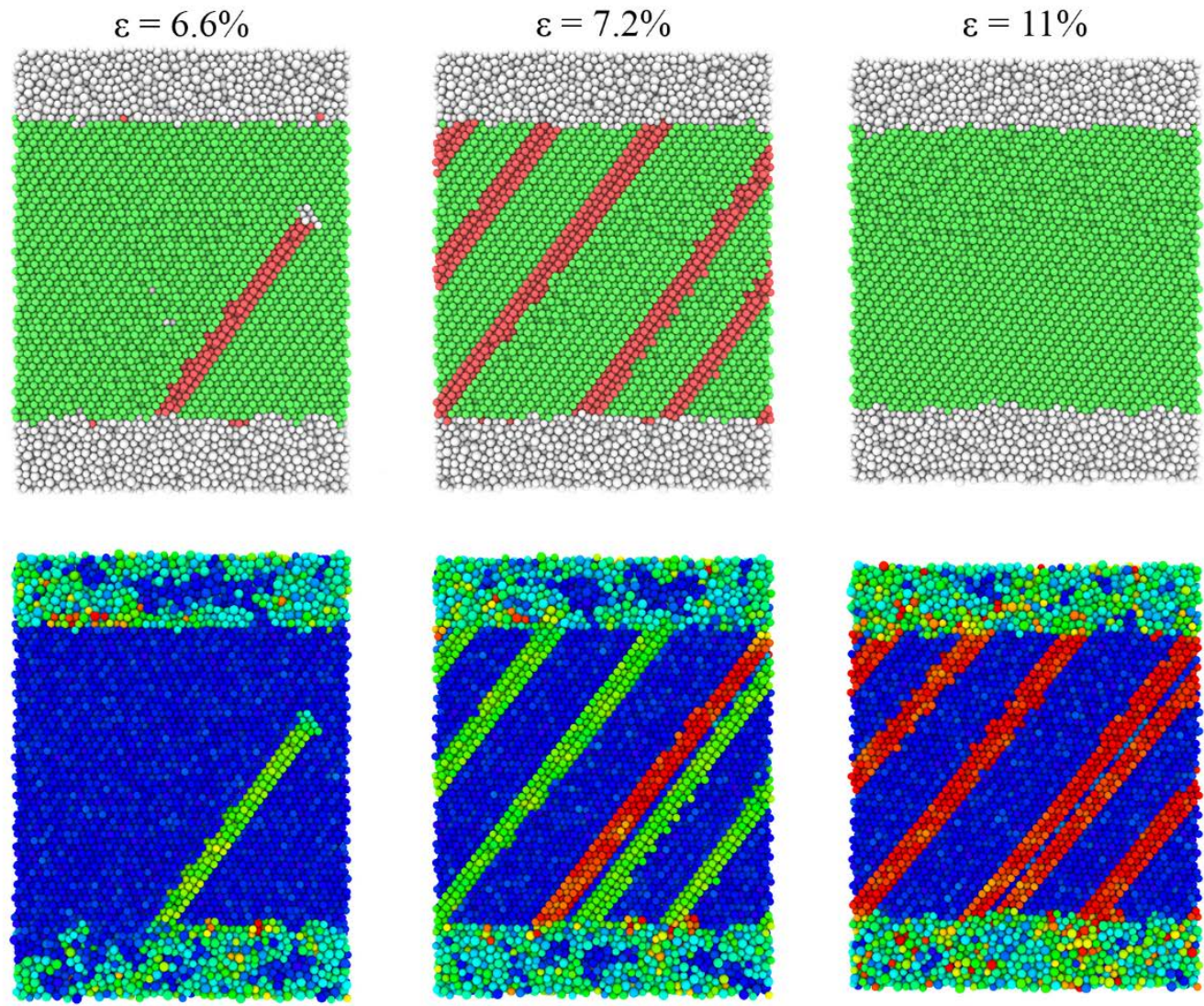

Slip Vector

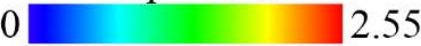

Figure 4: Deformation snapshots of the SC-NL structure viewed along the [110] direction at $6.6 \%, 7.2 \%$, and $11 \%$ strain colored by the CNA value (upper) and slip vector (lower). Partial and full dislocations emitted from the ACI traverse the (111) plane and are absorbed at the adjacent ACI. (The reader is referred to the web version of this article for interpretation of references to color.) 
$\varepsilon=3.2 \%$
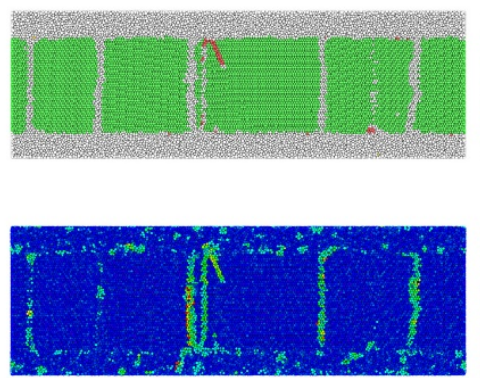

$\varepsilon=7.2 \%$
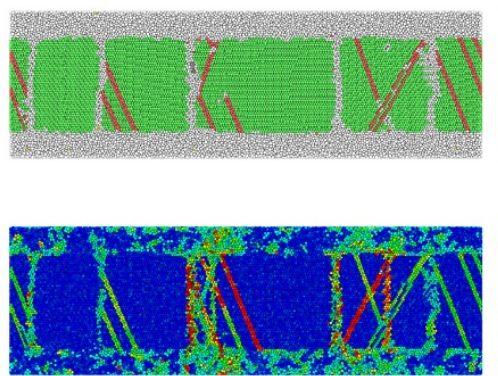

Slip Vector $\varepsilon=10.8 \%$
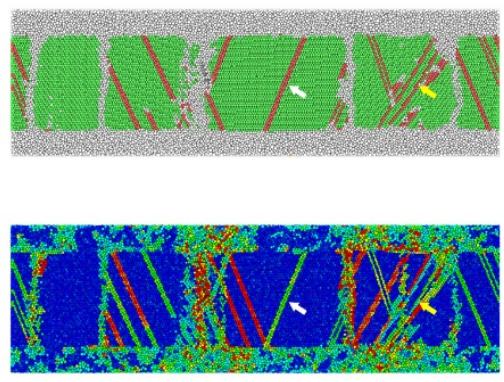

2.55

Figure 5: Deformation snapshots of the CN-NL structure viewed in the $\mathrm{x}-\mathrm{z}$ plane at 3.2\%, 7.2\%, and $10.8 \%$ strain colored by the CNA value (upper) and slip vector (lower). Dislocation activity is mediated by both the ACIs and grain boundaries, and results in dislocations propagating along multiple slip planes. (The reader is referred to the web version of this article for interpretation of references to color.) 


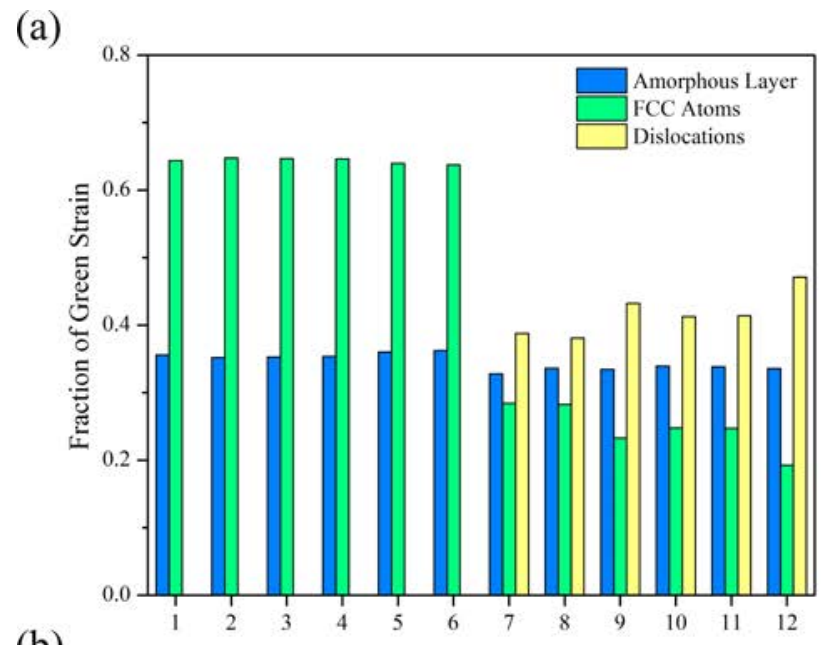

(b)

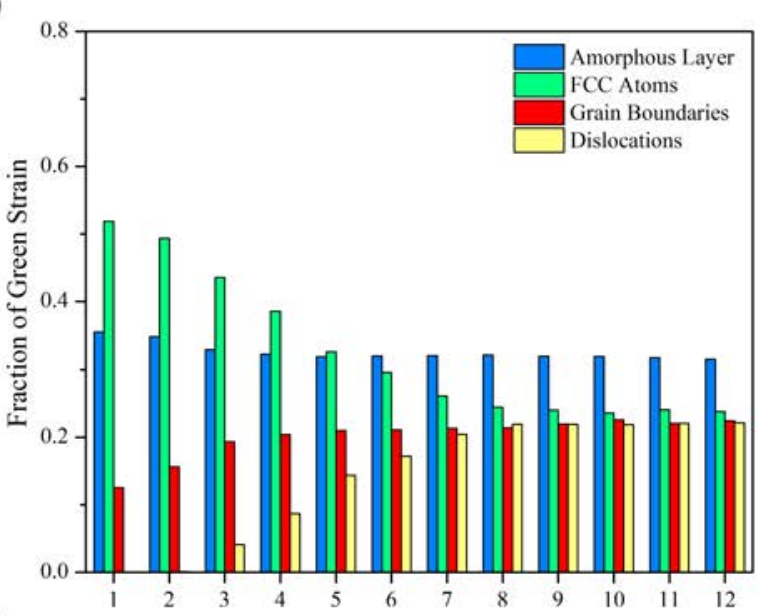

(c)

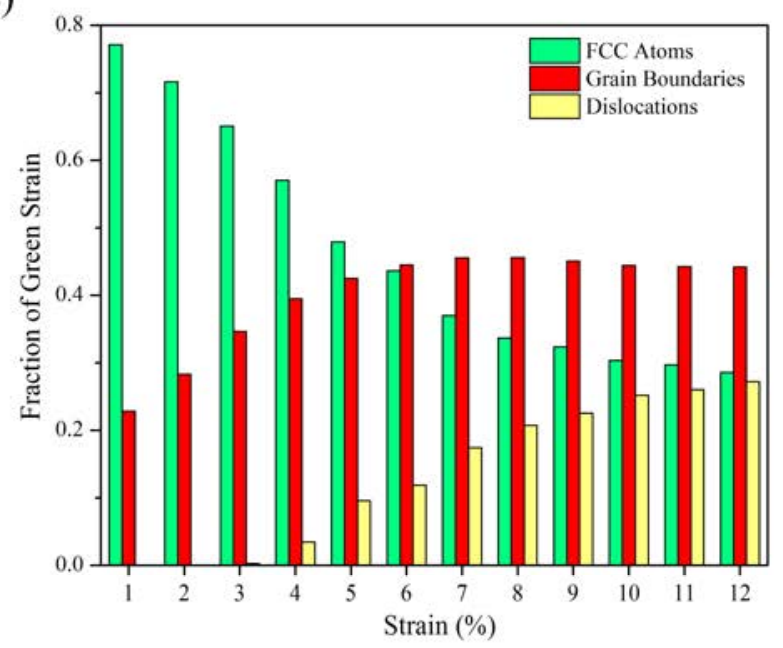

Figure 6: Strain accommodation maps for the (a) SC-NL, (b) NC-NL, and (c) NC-Cu structures that delineate the contribution of the amorphous layer, FCC atoms, grain boundaries, and dislocations to deformation. The series order in the legend aligns with the bar order at each strain from left-to-right. 
(a)
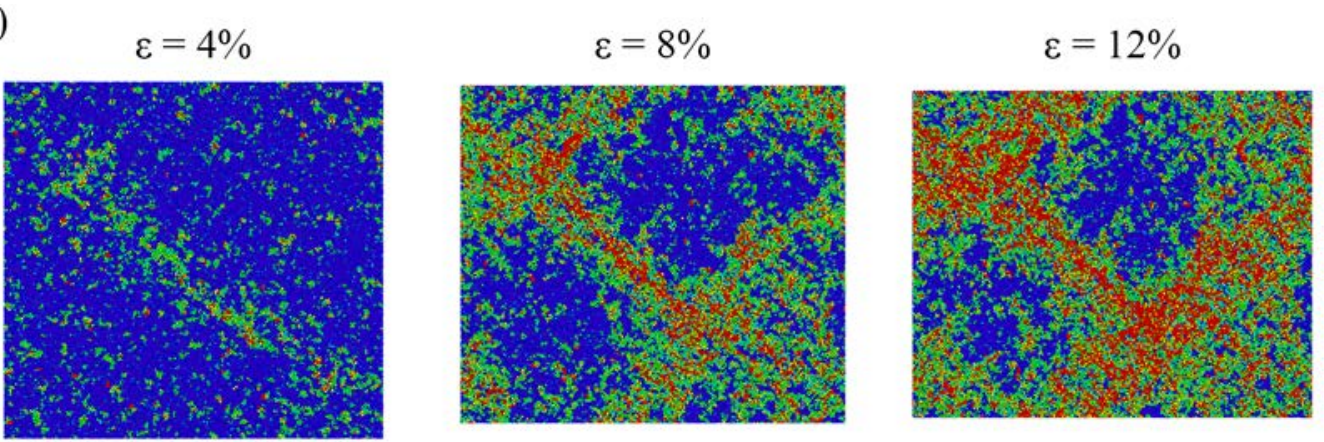

(b)
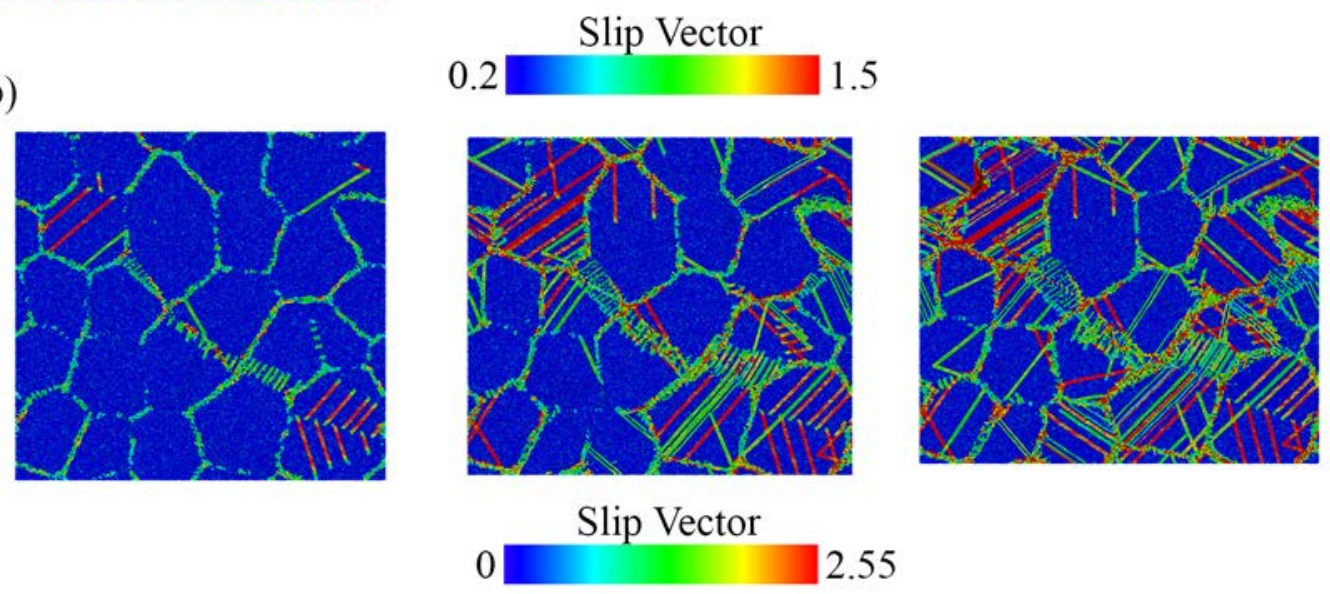

Figure 7: Deformation snapshots from the CN-NL sample viewed in the $x-y$ plane (i.e. topdown) of the (a) amorphous and (b) crystalline layers at strains of $4 \%, 8 \%$, and $12 \%$ with atoms colored according to the slip vector value. The alignment of dislocation slip bands with shear bands demonstrates the interaction of the disparate deformation mechanisms across the ACI. (The reader is referred to the web version of this article for interpretation of references to color.) 
(a)

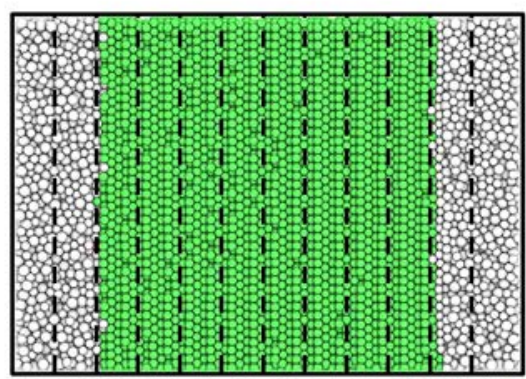

$\begin{array}{llllllllllllllll}\text { S1 } & \text { S2 } & \text { S3 } & \text { S4 } & \text { S5 } & \text { S6 } & \text { S7 } & \text { S8 } & \text { S9 } & \text { S10 } & \text { S11 } & \text { S12 }\end{array}$

(c)

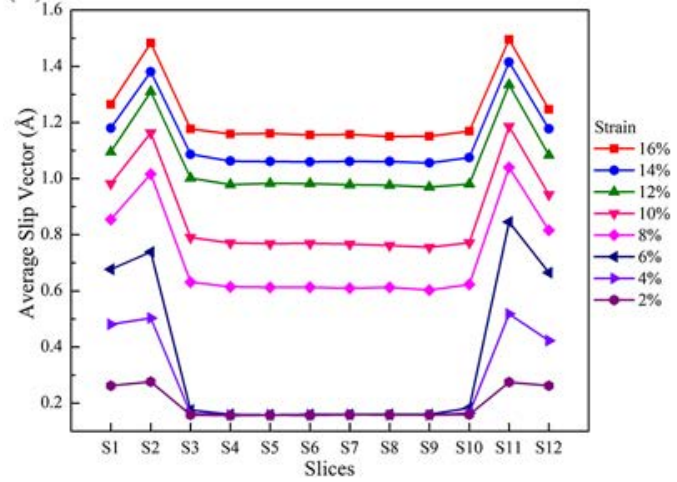

(b)

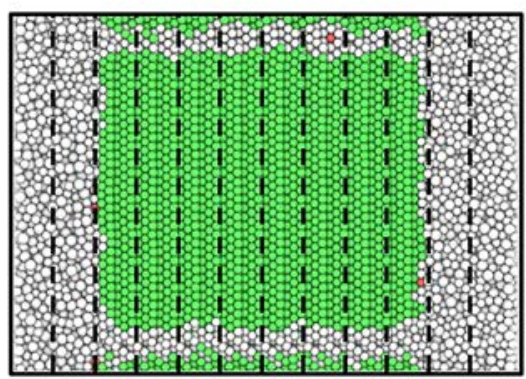

$\begin{array}{llllllllllllllll}\text { S1 } & \text { S2 } & \text { S3 } & \text { S4 } & \text { S5 } & \text { S6 } & \text { S7 } & \text { S8 } & \text { S9 } & \text { S10 } & \text { S11 } & \text { S12 }\end{array}$

(d)

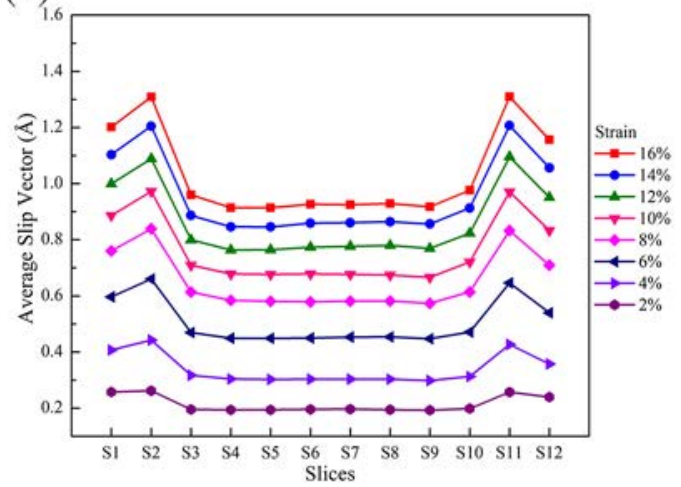

Figure 8: Orientation and location of the average slip vector slices for the (a) SC-NL and (b) CN-NL structures; the snapshots are colored according to CNA value and rotated $90^{\circ}$ relative to Figures 4 and 5. The average slip vector determined for each slice as a function of the applied strain for the (c) SC-NL and (b) CN-NL. (The reader is referred to the web version of this article for interpretation of references to color.) 

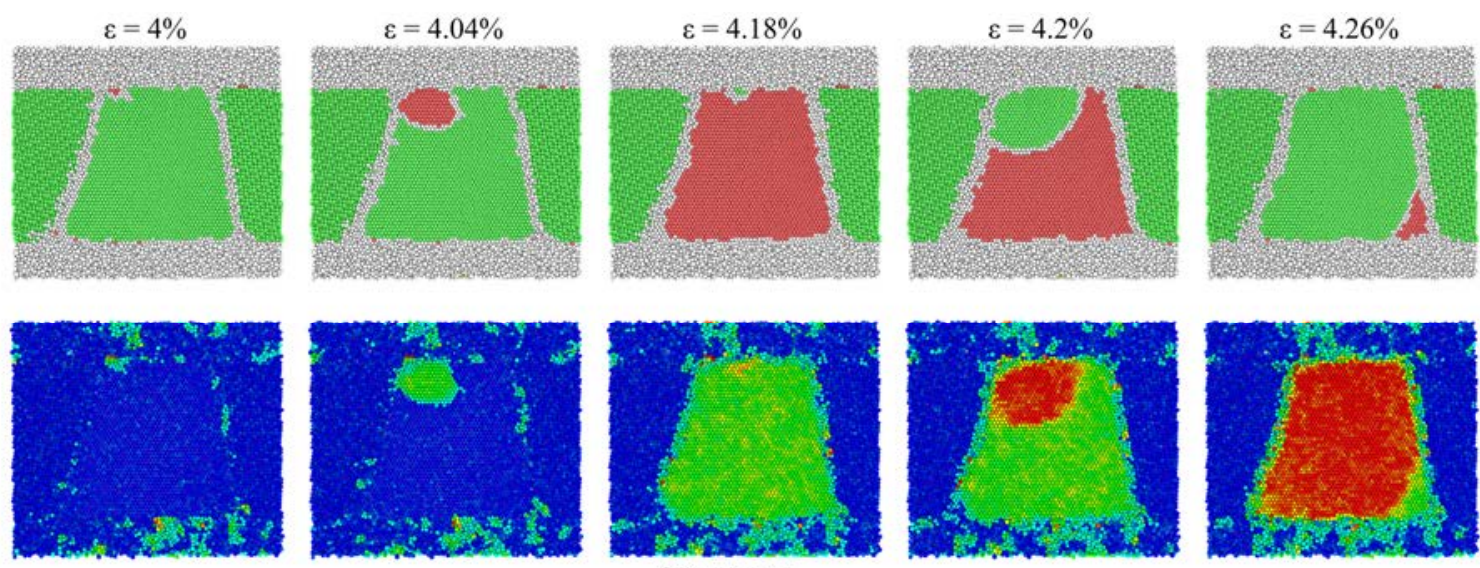

Slip Vector

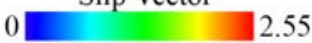

Figure 9: Deformation snapshots illustrating the evolution of a dislocation on the (11̄1) slip plane colored via the CNA values (upper) and slip vector (lower) between 4.0 and $4.26 \%$ strain. The dislocation nucleates from the intersection of a grain boundary with the upper ACI, propagates across the grain, and is absorbed into the amorphous layer across the lower ACI. (The reader is referred to the web version of this article for interpretation of references to color.) 
(a)

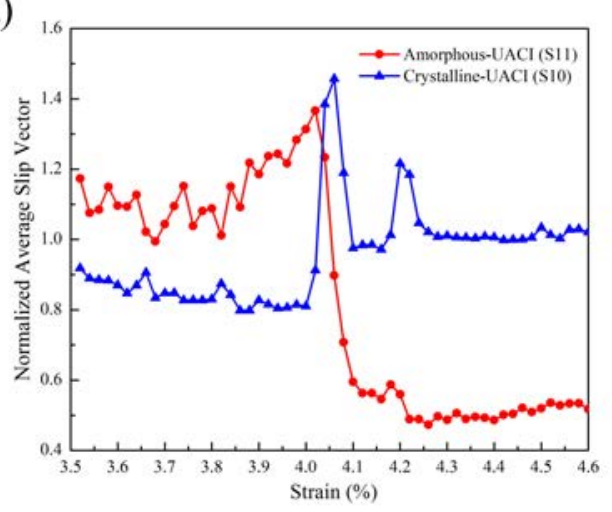

(b)

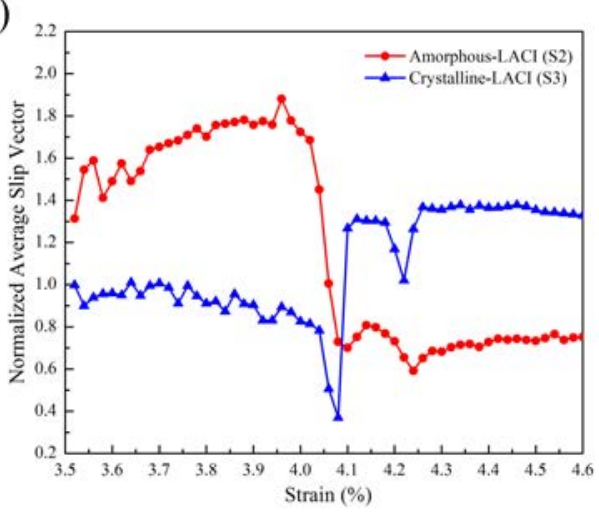

Figure 10: Normalized average slip vector as a function of strain for the (11 $\overline{1})$ slip event depicted in Figure 9. Trends are presented for amorphous and crystalline slices immediately adjacent to (a) the upper ACI (UACI) for slices S11 and S10, and (b) the lower ACI (LACI) for slices S2 and S3. 
(a)

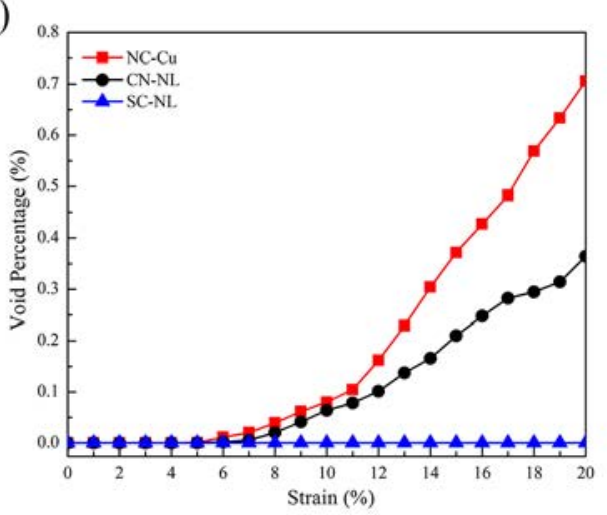

(c)

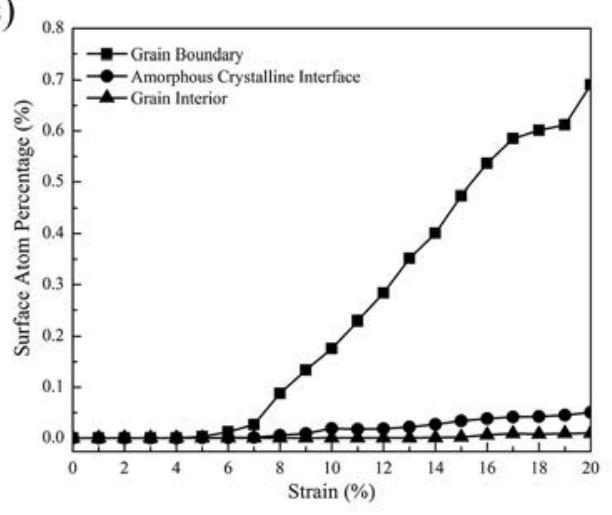

(b)

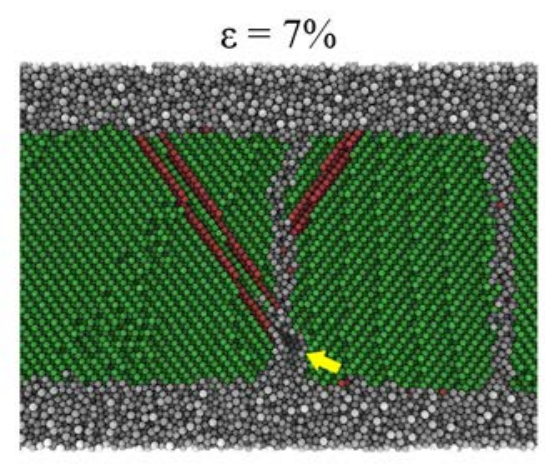

(d)

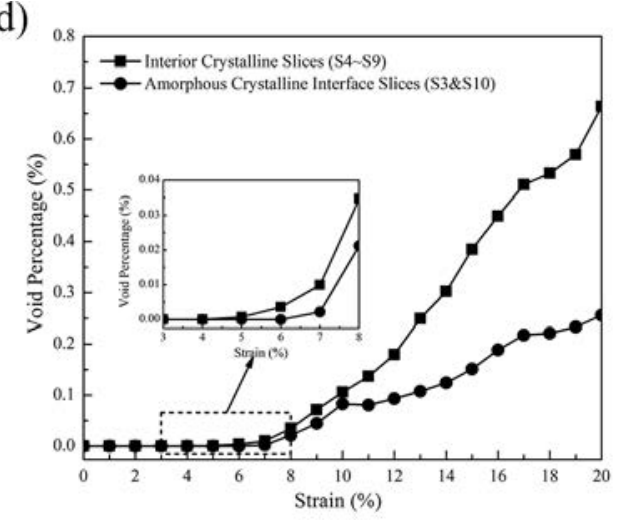

Figure 11: (a) Overall void volume fraction (in percentage) for each structure as a function of the strain. (b) Formation of a grain boundary nanovoid at 7\% strain in the CN-NL structure upon intersection of a leading partial dislocation with the grain boundary as highlighted by the yellow arrow; atoms are colored via their CNA value. (c) The fraction of surface atoms located in the grain interior, $\mathrm{ACI}$, and grain boundaries in the CN-NL as a function of strain. (d) Void fractions (in percentage) in the crystalline layer of the CN-NL structure where slices directly adjacent to the ACIs (S3 \& S10) are distinguished from those in the interior of the crystalline region (S4 S9). (The reader is referred to the web version of this article for interpretation of references to color.) 